\title{
Analytic Tools for Evaluating Variability of Standard Errors in Large-Scale Establishment Surveys
}

\author{
MoonJung Cho ${ }^{1}$, John L. Eltinge ${ }^{1}$, Julie Gershunskaya ${ }^{2}$, and Larry Huff ${ }^{2}$
}

\begin{abstract}
Large-scale establishment surveys often exhibit substantial temporal or cross-sectional variability in their published standard errors. This article uses a framework defined by survey generalized variance functions to develop three sets of analytic tools for the evaluation of these patterns of variability. These tools are for (1) identification of predictor variables that explain some of the observed temporal and cross-sectional variability in published standard errors; (2) evaluation of the proportion of variability attributable to the abovementioned predictors, equation error and estimation error, respectively; and (3) comparison of equation error variances across groups defined by observable predictor variables. The primary ideas are motivated and illustrated by an application to the U.S. Current Employment Statistics program.
\end{abstract}

Key words: Degrees of freedom; design effect; generalized variance function (GVF); U.S. Current Employment Statistics program.

\section{Introduction: Temporal and Cross-Sectional Variability of Published Standard Errors}

Large-scale establishment surveys often exhibit substantial temporal or cross-sectional variability in their published standard errors or relative standard errors. To illustrate, consider a set of domains $j$ and periods $t, j=1, \ldots, J ; t=1, \ldots, T$; let $\theta_{j t}$ be a finite population parameter for domain $j$ at time $t$; let $\hat{\theta}_{j t}$ be the associated design-based point estimator; let $\hat{V}_{p}\left(\hat{\theta}_{j t}\right)$ be an estimator of the design variance of $\hat{\theta}_{j t}$; and define the associated estimated standard errors

$$
s\left(\hat{\theta}_{j t}\right)=\left\{\hat{V}_{p}\left(\hat{\theta}_{j t}\right)\right\}^{1 / 2}
$$

and relative standard errors

$$
r\left(\hat{\theta}_{j t}\right)=\frac{s\left(\hat{\theta}_{j t}\right)}{\hat{\theta}_{j t}} .
$$

Throughout this article, the subscript " $p$ " denotes an expectation or variance evaluated with respect to the sample design.

1 U.S. Bureau of Labor Statistics-Office of Survey Methods Research, PSB 19502 Massachusetts Ave. N.E., Washington, DC, 20212, U.S.A. Emails: Cho.Moon@bls.gov, Eltinge.John@bls.gov

${ }^{2}$ U.S. Bureau of Labor Statistics-Office of Employment and Unemployment Statistics, Washington, DC, U.S.A. Emails: Gershunskaya.Julie@bls.gov and Huff.Larry@bls.gov

Acknowledgment: The authors thank Ken Robertson for many helpful discussions of the CES and the Associate Editor for the insightful and constructive suggestions. The views expressed in this article are those of the authors and do not necessarily reflect the policies of the U.S. Bureau of Labor Statistics. 
Variability of $s\left(\hat{\theta}_{j t}\right)$ and $r\left(\hat{\theta}_{j t}\right)$ can have a substantial practical effect on data users. Consequently, it is important for survey management to have diagnostic tools to assess this variability. For that assessment, four sources are of primary interest:

(A) Temporal or cross-sectional differences in the true design variances that are attributable to changes in factors that can be controlled (to some extent). For example, let $n_{j t}$ equal the realized sample size for domain $j$ at time $t$. If the variability in $s\left(\hat{\theta}_{j t}\right)$ or $r\left(\hat{\theta}_{j t}\right)$ were considered large enough to be problematic, and if it were attributable primarily to variability in $n_{j t}$, then one could consider a design modification that would reduce variability in $n_{j t}$ values.

(B) Differences in the true design variance $V_{p}\left(\hat{\theta}_{j t}\right)$ that are attributable to changes in factors that can be observed (or estimated from available data) but not controlled. For example, the true design variance and relative variance may be functions of estimable parameters of the underlying finite population, for example, functions of the elementlevel population variance, and of the true population parameter $\theta_{j t}$.

(C) Differences in the true design variance $V_{p}\left(\hat{\theta}_{j t}\right)$ that are attributable to factors that are neither controllable, nor observable, nor readily estimable. Examples include changes in $V_{p}\left(\hat{\theta}_{j t}\right)$ that arise from short-term local changes in economic conditions.

(D) Sampling variability of the variance estimator $\hat{V}_{p}\left(\hat{\theta}_{j t}\right)$. For surveys to which case (D) applies, one may wish to consider using an alternative to the current variance estimator.

Issues (A) through (D) can arise for both household and establishment surveys. For establishment surveys, these issues can be especially interesting due to two factors. First, many survey variables are approximately continuous and have heavily skewed population distributions. For example, in the establishment survey application considered below, individual employment counts range from single digits to tens of thousands, but most population units had counts in the single or double digits. Second, initiation of new sample units can be expensive and time consuming. To address these issues, many establishment surveys use a panel structure, and realized sample sizes may vary due to the effects of slow sample initiation, as well as attrition. This in turn may lead to increased variability in the true design variances.

The remainder of this article develops methods for exploration of sources (A) through (D) outlined above. These methods are based on relatively simple parametric models for the regression of $\ln \left(\hat{V}_{p}\left(\hat{\theta}_{j t}\right)\right)$ on predictor variables associated with sources (A) and (B). Such regression models may be viewed as extensions of generalized variance function models developed previously in the sample survey literature. Specifically, Section 2 provides a brief introduction to a case study based on the U.S. Current Employment Statistics Program. Section 3 develops some notation for the predictors, coefficients and error terms that will be important for these generalized variance function (GVF) extensions, and outlines estimation and inference methods for the applicable GVF models. Section 4 considers sources (A) and (B) through evaluation of the extent to which variability in $s\left(\hat{\theta}_{j t}\right)$ may be associated with variability in observed predictors. Section 5 applies the main ideas of Sections 3 and 4 to the CES example introduced in Section 2; and also uses estimators of the equation-error variance to evaluate source (C). Section 6 reviews the main ideas of this article; discusses conditions under which source (D) may also be of practical importance; and considers several possible extensions of the methods developed here. 


\section{An Example: Monthly Estimation from the U.S. Current Employment Statistics Program}

This article was motivated by variability in the direct standard errors computed for the U.S. Current Employment Statistics (CES) survey. The CES survey collects data each month on employment, hours, and earnings from a sample of nonagricultural establishments. The sample includes approximately 140,000 businesses and government agencies, covering around 440,000 individual worksites. Approximately 55,000 new sample units are enrolled in the CES survey each year to account for the establishment of new firms and to rotate a portion of the sample. When firms are rotated into the sample, they are retained for two years or more. The active CES sample includes approximately one third of all nonfarm payroll employees.

The CES design uses a stratified simple random sample of unemployment insurance (UI) accounts. A UI account is a cluster that may contain single or multiple establishments. The sample strata or subpopulations are defined by state, industry, and employment size class, yielding a state-based design. For a given sample size per state, sampling rates for each stratum are determined through optimum allocations to minimize the overall sampling error variance of the estimated statewide total private employment. All data on employment, hours, and earnings for the nation and for states and areas are classified in accordance with the 2007 North American Industry Classification System (NAICS). See the BLS Handbook of Methods (U.S. Bureau of Labor Statistics 2011, ch. 2), Butani et al. (1997) and Werking (1997) for further details.

CES uses a "weighted link relative estimator" of the employment in domain $j$ for month $t$. This estimator is computed as the product

$$
\hat{y}_{j t}=x_{j 0} \hat{R}_{j t},
$$

where $x_{j 0}$ is the known Quarterly Census of Employment and Wages (QCEW) employment total for all establishments in domain $j$ for the benchmark month $0 ; \hat{y}_{j t}$ is an estimator of the unknown true employment total for domain $j$ in month $t$; and $\hat{R}_{j t}$ is an estimator of the relative employment growth that took place from benchmark month 0 to the current month $t$ as detailed in BLS Handbook of Methods (U.S. Bureau of Labor Statistics 2011) and Gershunskaya and Lahiri (2005). For the current article, the domains of interest are 14 large industries described in Table 1.

For a given reference month, the CES publishes estimates labeled "first closing", "second closing" and "third closing"; the second and third closing estimates use additional information from respondents not available for the first-closing estimates at the time of production. All results reported in this article are for sample sizes, point estimates and variance estimates for the third-closing data. For an additional discussion of the first, second and third closing for the CES, see Copeland and Valliant (2007).

The CES publishes many estimates of employment changes over time periods of varying lengths. However, this article will focus attention on only three distinct estimators: total employment, $\hat{y}_{j t}$, one-month change, $\hat{y}_{j t}-\hat{y}_{j, t-1}$, and one-month relative change $\left(\hat{y}_{j, t-1}\right)^{-1} \hat{y}_{j t}$. In the discussion below, the generic term $\hat{\theta}_{j t}$ may represent any of these three estimators. In addition, the estimates $\hat{y}_{j t}, \hat{y}_{j t}-\hat{y}_{j, t-1}$ and $\left(\hat{y}_{j, t-1}\right)^{-1} \hat{y}_{j t}$ and their associated variance estimates are computed for each month $t=1,2, \ldots, 20$. These months 
Table 1. Description of industries

\begin{tabular}{lll}
\hline Industry & Description & Classification \\
\hline 1 & Mining and logging & Goods-producing \\
2 & Construction & Goods-producing \\
3 & Durable goods manufacturing & Goods-producing \\
4 & Nondurable goods manufacturing & Goods-producing \\
5 & Wholesale trade & Service-providing \\
6 & Retail trade & Service-providing \\
7 & Transportation and warehousing & Service-providing \\
8 & Utilities & Service-providing \\
9 & Information & Service-providing \\
10 & Financial activities & Service-providing \\
11 & Professional and business services & Service-providing \\
12 & Education and health services & Service-providing \\
13 & Leisure and hospitality & Service-providing \\
14 & Other services & Service-providing \\
\hline
\end{tabular}

correspond to March of a given year through October of the subsequent year. However, for a specified benchmark month 1, only results from the corresponding months 8 through 19 (October through the following September) are included in official third-closing publications. Consequently, all results presented in this article are based on data from these reference months 8 through 19 .

Figure 1 presents boxplots of monthly realized sample sizes $n_{j t}$ for the fourteen industries in the years 2005-2010. For CES national-level estimators, variance estimators are computed using balanced half-sample (BHS) methods, with Fay factors (Judkins 1990). These estimators include stratum-level finite population corrections. This article will use the symbols $\hat{V}_{p j t}$ to denote the BHS variance estimator for domain $j$ and time $t$.

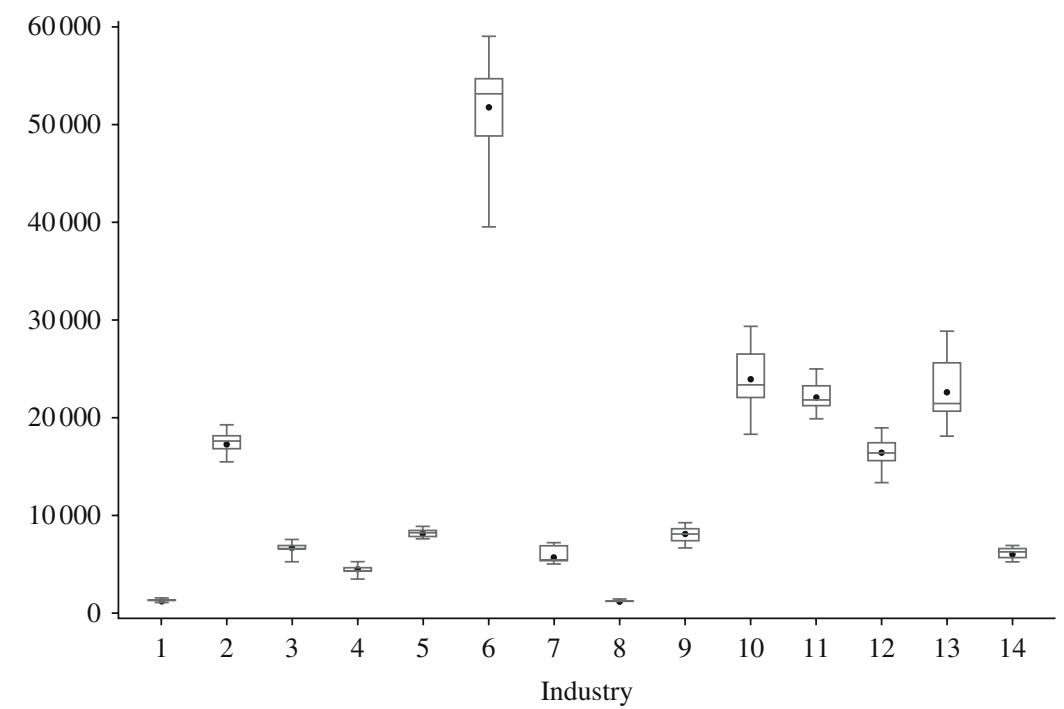

Fig. 1. Boxplots of the monthly numbers of responding sample units $\left(n_{j t}\right)$ from years $2005-2010$ for each of industries 1 though 14 
Figure 2 presents boxplots of the natural logarithms of the BHS variance estimates, $\ln \left(\hat{V}_{p j t}\right)$ for monthly total employment in the specified industries. Note that log-scale differences $\ln \left(\hat{V}_{1}\right)-\ln \left(\hat{V}_{2}\right)=1.5,2.0$ and 3.0 correspond to variance ratios $\left(\hat{V}_{1} / \hat{V}_{2}\right)$ equal to $4.5,7.4$ and 20.1, respectively, and standard error ratios, $\left(\hat{V}_{1} / \hat{V}_{2}\right)^{1 / 2}$ equal to $2.1,2.7$ and 4.5 , respectively. Consequently, the log-scale differences displayed in Figure 2 correspond to substantial differences on the standard error and variance scales.

To explore these patterns of variability at an industry level, Figure 3 presents a time plot of $n_{j t}$ for construction; Figures 4 and 5 present the corresponding time plots of $\ln \left(\hat{V}_{p j t}\right)$ for total employment and one-month change respectively. Figures 3 displays "saw-tooth" patterns due to the periodic initiation of new units and continuing attrition of current units. In addition, the numbers of respondents $n_{j t}$ generally show a marked increase between October and November of a given year. Similar plots were produced for other industries such as retail trade but are not shown in the article.

Furthermore, for a given benchmark year, the BHS variance estimator of total employment tends to increase across months, that is, the variance increases as the reference month moves farther away from the benchmark month. However, temporal trends with respect to months are considerably less pronounced in cases of one-month change and one-month relative change estimators.

\section{Model Development, Estimation and Inference for Generalized Variance Functions}

\subsection{General Models for the True Design Variance}

Due to the temporal variability in the standard errors computed from the BHS method, $s\left(\hat{\theta}_{j t}\right)=\left\{\hat{V}_{p}\left(\hat{\theta}_{j t}\right)\right\}^{1 / 2}$, the CES program does not currently publish the values of $s\left(\hat{\theta}_{j t}\right)$ as such. Instead, it publishes temporal medians of these standard errors. However, the CES

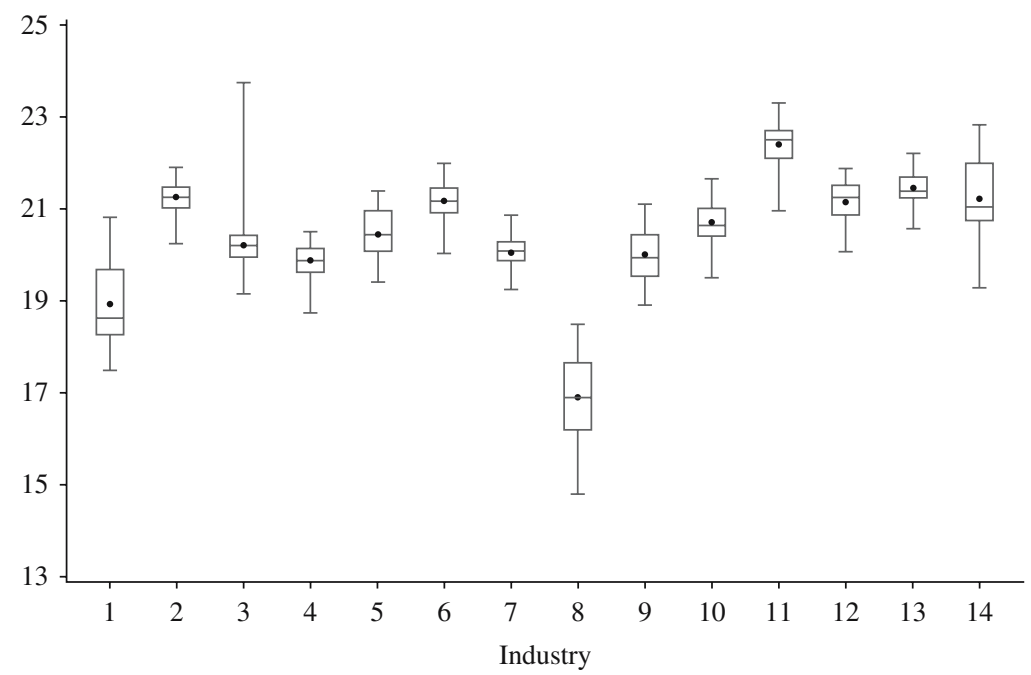

Fig. 2. Boxplots of $\ln \left(\hat{V}_{p j t}\right)$ for monthly estimates of total employment from years 2005-2010, separately for industries $1-14$ 


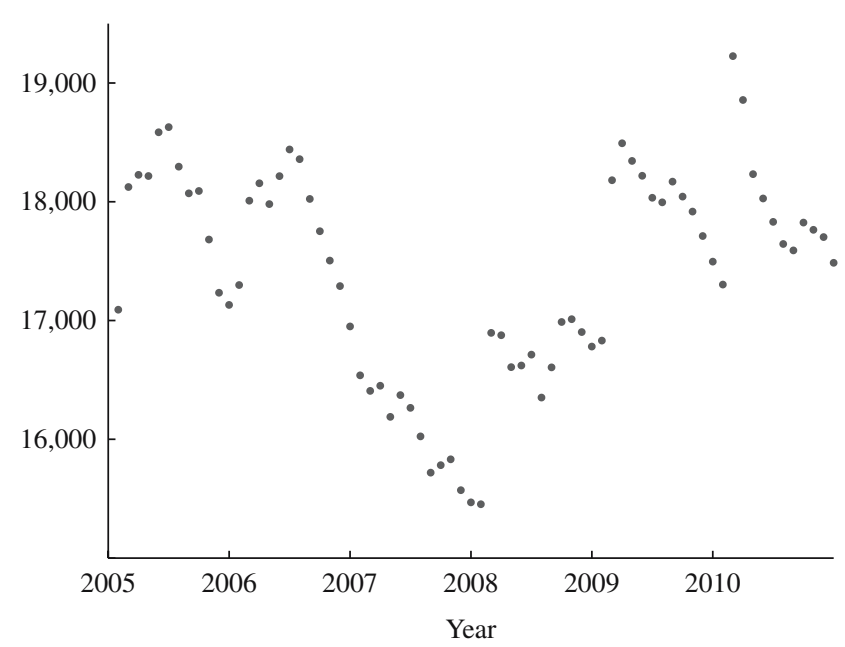

Fig. 3. Number of responding sample units $\left(n_{j t}\right)$ across years: construction (monthly realized sample sizes for October 2005 through September 2011)

program is interested in exploring the reasons for variability of $s\left(\hat{\theta}_{j t}\right)$, and in using the results of that exploration to develop alternative variance estimators.

To begin that exploratory study, let $X_{A_{j t}}$ be a vector of predictors that can be observed and controlled; let $X_{B_{j t}}$ be an additional vector of predictors that can be observed or estimated but not controlled; define $X_{j t}=\left(X_{A_{j t}}, X_{B_{j t}}\right)$; define $V_{p j t}=V_{p}\left(\hat{\theta}_{j t}\right)$; and consider a general model

$$
\ln \left(V_{p j t}\right)=g\left(X_{j t}, \gamma\right)+q_{j t}^{*}
$$

where $q_{j t}^{*}$ is a univariate "equation error" with a mean equal to zero, and $\gamma$ is a $b$-dimensional vector of variance function parameters. Note especially that $q_{j t}^{*}$ represents

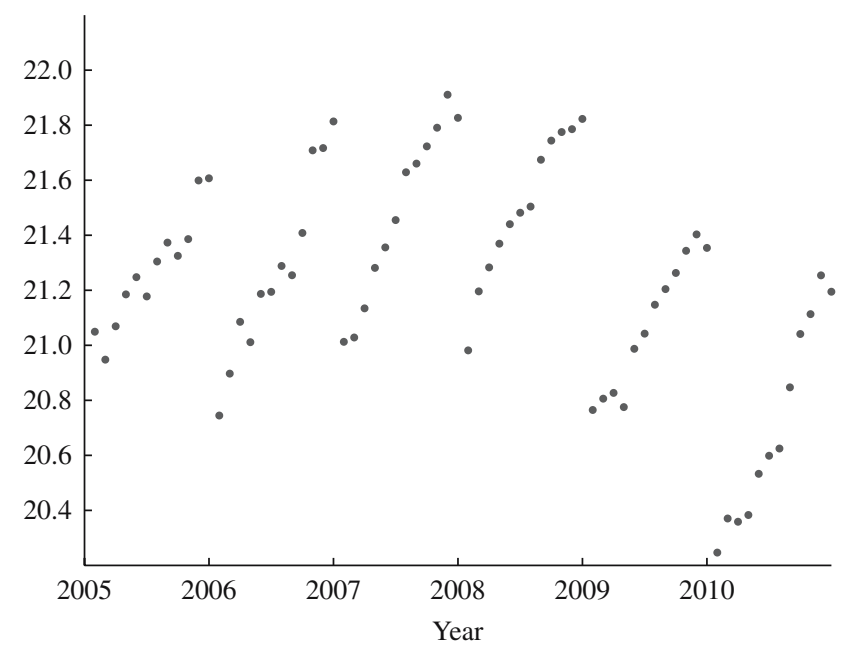

Fig. 4. Plot of $\ln \left(\hat{V}_{p j t}\right)$ of total employment across years: construction industry (estimates for October 2005 through September 2011) 


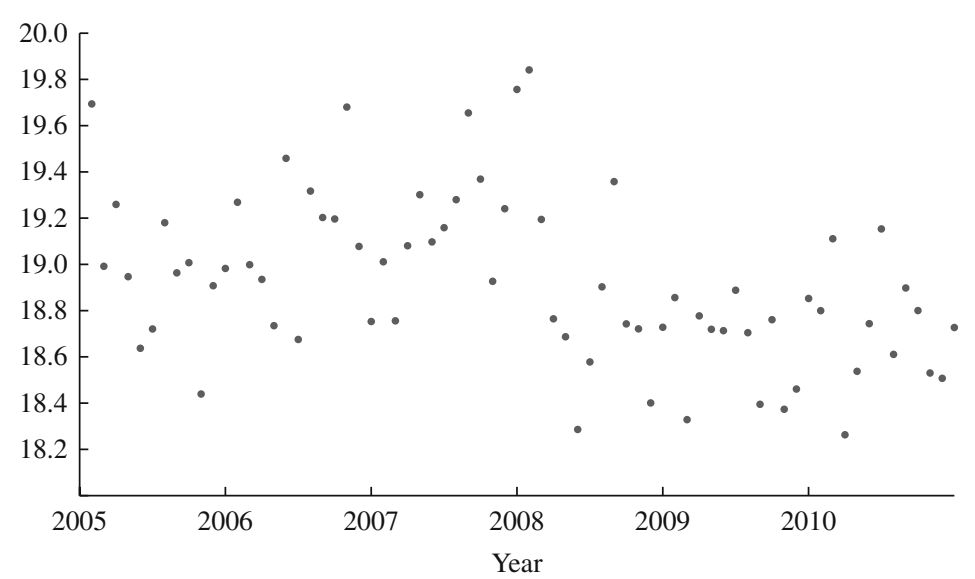

Fig. 5. Plot of $\ln \left(\hat{V}_{\text {pjt }}\right)$ of One-month change across years: construction (estimates for October 2005 through September 2011)

the deviation of logarithm of the true design variance $V_{p j t}$ from its modeled value $g\left(X_{j t}, \gamma\right)$. Model (2) may be considered a type of generalized variance function, as developed in Johnson and King (1987), Valliant (1987), O’Malley and Zaslavsky (2005), Wolter (2007, sec. 7.2), Cho et al. (2002), Cho et al. (2014) and references cited therein. Some previous authors (e.g., Johnson and King 1987) have also developed generalized variance function models on logarithmic scales. Use of a logarithmic scale converts multiplicative relationships to linear relationships, and reduces the effects of extreme values.

Much of the GVF literature has focused on the variances of point estimators $\hat{\theta}_{j t}$ for population proportions or population totals related to a binary outcome variable; and has tended to emphasize predictors $X_{B_{j t}}$. In addition, much of this literature has used $\theta_{j t}$ as one component of the predictor vector $X_{B_{j t}}$. The current article, however, considers the more complex setting in which the point estimator of interest depends primarily on survey variables that are not binary; it will use predictors $X_{A_{j t}}$ and $X_{B_{j t}}$ that are not necessarily related to the value of $\theta_{j t}$, but are related to important features of the sample design or estimation process.

On a logarithmic scale, one example of Model (2) is

$$
\ln \left(V_{p j t}\right)=\gamma_{0}+\gamma_{A} X_{A_{j t}}+\gamma_{B} X_{B_{j t}}+q_{j t}^{*}
$$

where $\gamma=\left(\gamma_{0}, \gamma_{A}, \gamma_{B}\right), \gamma_{0}$ is univariate, $\gamma_{A}$ is $1 \times b_{A}, \gamma_{B}$ is $1 \times b_{B}, X_{A_{j t}}$ is $b_{A} \times 1, X_{B_{j t}}$ is $b_{B} \times 1, b=1+b_{A}+b_{B}$ and $q_{j t}^{*}$ is a random variable with mean equal to zero and variance equal to $\sigma_{q_{j t}^{*}}^{2}$.

Before exploring specific forms of the Models (2) and (3), it is useful to add four comments on the conceptual basis for generalized variance functions. First, these functions are intended to approximate the true variances of $\hat{\theta}_{j t}$, considered over the set defined by $j=1, \ldots, J$ and $t=1, \ldots, T$, and averaging over all of the sources of random variability considered important for understanding the properties of $\hat{\theta}_{j t}$. In some of the original GVF literature, the only source considered was traditional sampling variability. However, in many cases, practical interest encompasses additional sources of variability, for example, the effects of nonresponse and measurement error. For these latter 
applications, one would need to define $V_{p j t}$ to include the relevant sources of both sampling and nonsampling error.

Second, practical fitting of Model (2) involves linear or nonlinear regression of the BHS variance estimators $\hat{V}_{p j t}$ on the corresponding predictors $X_{j t}$. Thus it is important for the BHS estimators $\hat{V}_{p j t}$ to be approximately unbiased for the variance terms $V_{p j t}$ of interest. For example, if interest centers on variance terms $V_{p j t}$ that include the effects of nonresponse, and of weighting adjustment or imputation used to construct $\hat{\theta}_{j t}$, then one would need to use BHS variance estimators $\hat{V}_{p j t}$ that incorporate these effects, for example, through Rao-Shao adjustments or multiple imputation. Similarly, if one intends to account for the effects of measurement errors on $\hat{\theta}_{j t}$, then it would be important to use initial estimators $\hat{V}_{p j t}$ that account for the combined effects of sampling error and measurement error, per Wolter (2007, app. D).

Third, similar comments apply to variance function models, such as Model (3), that are fit following a nonlinear transformation. For these cases, it is important to account for transformation effects in discussion of unbiased estimation. Valliant (1987) provides a rigorous conceptual basis for generalized variance functions under some specific superpopulation models.

Fourth, the choice of approximate predictors $X_{A_{j t}}$ and $X_{B_{j t}}$ will depend on specific features of a given application. Important criteria include availability of the predictors at the appropriate level of aggregation; potential relevance of the predictors, based on features of the sampling and estimation process; empirical assessment of the statistical significance of the coefficients of the predictors in specific models; and related diagnostics for the goodness-of-fit for the variance function model when specific predictors are included. The remainder of this article explores these ideas in additional detail.

\subsection{Point Estimation and Variance Estimation for Coefficients}

For several versions of a Model (3), we computed estimators $\hat{\gamma}$ of the coefficients $\gamma$ through ordinary least squares (OLS) regression of $\ln \left(\hat{V}_{p j t}\right)$ on the corresponding vector of predictors. In keeping with Valliant (1987), one could consider alternative estimators of $\gamma$ based on weighted least squares methods, with weights proportional to the inverses of preliminary estimators of variances of the error terms in Model (3). However, exploratory application of this idea to the CES data encountered issues with numerical stability; see Section 6 for related comments.

In addition, practical work with GVFs can require one to identify groups of estimators $\hat{\theta}_{j t}$ for which a common set of coefficients $\gamma$ may be used. Some authors have addressed this need through qualitative identification of estimators with similar design or population features; for an example see Wolter $(2007,276)$. To complement this qualitative approach, it is useful to produce estimators of the variance of the coefficient vector estimator $\hat{\gamma}$, and to carry out significance testing for homogeneity of the coefficients across groups. For example, Subsection 5.2 will present results on comparison of coefficients across years and across industry groups. For this goal, we obtained an estimator $\hat{V}_{p}(\hat{\gamma})$ of the variance of the approximate distribution of $\hat{\gamma}$ from an extension of standard estimating equation approaches for complex-survey estimators (Binder 1983). Details of the estimating equation formulation for GVF cases and its applications were provided in Cho et al. (2014). This formulation 
accounted directly for the features of the sample design and the point estimators $\hat{\theta}_{j t}$. In the CES example, the dependent variables $\hat{V}_{p j t}$ may be strongly correlated across months, due to the form of the weighted link relative estimators as well as the use of a rotation sample design. However, sampling is essentially independent across domains. Thus we decomposed the estimating equation into sums of terms across independent domains. Based on this designadjusted variance estimator for $\hat{\gamma}$, Cho et al. (2014) showed that standard (unadjusted) variance estimates for $\hat{\gamma}$ may be much smaller than the unbiased estimates. Consequently, it is important to use design-adjusted estimators, $\hat{V}_{p}(\hat{\gamma})$, in inference for $\gamma$.

\subsection{Models for Variance Estimation Error}

Now consider again the temporal and cross-sectional variability in standard errors discussed in Section 1. Within the framework defined by Model (3), Sources (A) and (B) correspond to the regression terms $\gamma_{A} X_{A_{j t}}$ and $\gamma_{B} X_{B_{j t}}$, respectively; and Source (C) corresponds to the equation error term $q_{j t}^{*}$. In addition, design features associated with Source (A) and the choice of a specific variance estimator $\hat{V}_{p j t}$ can both have an effect on the sampling errors defined by the differences

$$
e_{j t}=\hat{V}_{p j t}-V_{p j t}
$$

for Source (D). Note especially that the sampling errors $e_{j t}$ are conceptually distinct from the equation errors $q_{j t}$ in Expression (2). Similar distinctions arise in other work with sampling errors and measurement errors. See, for example, Fuller (1987). In some cases, one may treat the distribution of the $e_{j t}$ terms as a rescaled and centered version of a chi-squared distribution on $d_{j t}$ degrees of freedom, that is,

$$
V_{p j t}^{-1} d_{j t} \hat{V}_{p j t}=V_{p j t}^{-1} d_{j t} e_{j t}+d_{j t} \sim \chi_{d_{j t}}^{2} .
$$

Some of the sample survey literature approximates $d_{j t}$ as the difference between the number of primary sampling units and the number of strata applicable to domain $j$ at time $t$. For some discussion of conditions under which this approximation may be appropriate, see Korn and Graubard (1990), Valliant and Rust (2010) and references cited therein. Our CES analyses will consider only estimators of national-level population parameters for relatively large industries. For such cases, the abovementioned computations lead to values of $d_{j t}$ greater than 100. Consequently, the current article will devote relatively limited attention to the sampling error terms $e_{j t}$.

\section{Differences Attributable to Variability in the Predictors $X_{j t}$; Equation Error; and Estimation Error}

In keeping with standard approaches to decomposition of sums of squares in regression (e.g., Draper and Smith 1998, ch. 6), one may decompose the variability of $\ln \left(\hat{V}_{p j t}\right)$ into four terms:

$S S_{A}$ : The sum of squared differences associated with controllable predictors $X_{A}$ $S S_{B \mid A}$ : The sum of squared differences associated with predictors $X_{B}$, after accounting for the controllable-predictor terms $X_{A}$ variability 
$S S_{Q}$ : The variability associated with equation error (sometimes called "lack of fit" error in the regression literature)

$S S_{P E}$ : The variability attributable to the random variability of $\ln \left(\hat{V}_{p j t}\right)$ conditional on $\ln \left(V_{p j t}\right)$ (sometimes called "pure error" in the regression literature)

For the CES national-level work, Subsection 3.3 noted that the $\hat{V}_{p j t}$ estimators are associated with relatively large "degrees of freedom" terms $d_{j t}$. Consequently, our analysis will use the assumption that the conditional variance $V_{p}\left[\ln \left(\hat{V}_{p j t}\right) \mid V_{p j t}\right]$ is approximately equal to zero. Note that $V_{p}\left[\ln \left(\hat{V}_{p j t}\right) \mid V_{p j t}\right]$ reflects the sampling variability of $\ln \left(\hat{V}_{p j t}\right)$ after conditioning on the true variance term $V_{p j t}$, and thus is essentially conditioning on the predictors $X_{j t}$ and the equation errors $q_{j t}^{*}$. Thus we will use the corresponding assumption that $S S_{P E}=0$. With this approximation, we have the decomposition of the "corrected total" sum of squares

$$
\begin{aligned}
S S C T & =\sum_{j=1}^{J} \sum_{t=1}^{T}\left\{\ln \left(\hat{V}_{p j t}\right)-L_{. .}\right\}^{2} \\
& =S S_{A}+S S_{B \mid A}+S S_{Q} \\
& =S S_{B}+S S_{A \mid B}+S S_{Q}
\end{aligned}
$$

where $L_{. .}=J^{-1} T^{-1} \sum_{j=1}^{J} \sum_{t=1}^{T} \ln \left(\hat{V}_{p j t}\right)$. In addition, for a full-model fit

$$
\ln \left(\hat{V}_{p j t}\right)=\gamma_{0}+\gamma_{A} X_{A_{j t}}+\gamma_{B} X_{B_{j t}}+q_{j t}^{*},
$$

the customary model $R^{2}$ equals the ratio

$$
(S S C T)^{-1}\left(S S_{A}+S S_{B \mid A}\right)=(S S C T)^{-1}\left(S S_{B}+S S_{A \mid B}\right) .
$$

Furthermore, for the partial model fit $\ln \left(\hat{V}_{p j t}\right)=\gamma_{0}+\gamma_{A} X_{A_{j t}}+q_{j t}^{*}$, the resulting model $R^{2}$ equals the ratio $(S S C T)^{-1} S S_{A}$. Similar comments apply to the partial model fit

$$
\ln \left(\hat{V}_{p j t}\right)=\gamma_{0}+\gamma_{B} X_{B_{j t}}+q_{j t}^{*}
$$

with model $R^{2}$ equal to $(S S C T)^{-1} S S_{B}$.

\section{Application to the U.S. Current Employment Statistics Program}

\subsection{Models from the Decomposition of the Design Variance}

To identify some potential predictors $X_{A}$ and $X_{B}$ for the CES application, recall that our sample consists of unemployment insurance accounts, which report nonzero employment for previous and current months. Let $n_{j t}$ be a number of responding UI accounts, $N_{j t}$ be a number of total UI accounts, and $S_{j t}^{2}$ be a finite population variance within the domain $j$ at time $t$. Then, we can express the variance of $\hat{y}_{j t}$ as a function of a design effect, $\Delta_{j t}$, for $\hat{y}_{j t}$. Ignoring the finite-population correction term, we write the variance of $\hat{y}_{j t}$, in terms of $\Delta_{j t}$ 
on the original variance scale:

$$
V_{p}\left(\hat{y}_{j t}\right)=\Delta_{j t}\left(n_{j t}^{-1} S_{j t}^{2} N_{j t}^{2}\right) .
$$

For some general background on design effects and their use in variance approximations, see, for example, Kish (1995), Park and Lee (2004) and references cited therein.

Note that Expression (9) uses the variance term $S_{j t}^{2}$, the finite population variance of the original employment counts $y_{j t}$. The design effect term $\Delta_{j t}$ incorporates all of the ratio estimator effects. In addition, for point estimators such as (1) that are based on estimators of cumulative growth from a benchmark month, $\Delta_{j t}$ may be an increasing function of $t$ (i.e., the design variance increases as the reference month moves further away from the benchmark month). For example, one could consider the approximation

$$
\Delta_{j t} \doteq \Delta t^{\alpha_{0}}
$$

where $\Delta$ is a common design effect term shared across domains $j$. Moreover, several authors have considered cases in which (sub)population variances are functions of associated (sub)population means or totals. For example, Cochran $(1977,243)$ discusses approximation of a finite population variance of an area unit as proportional to a positive power of the size of that unit. Similarly, Box-Cox transformations are often based on the assumption of a power relationship between the means and variances of sets of observations. Application of this idea to the CES leads to the approximation

$$
S_{j t}^{2} N_{j t}^{2} \doteq \alpha_{1}\left(x_{j 0}\right)^{\alpha_{2}}
$$

where $\alpha_{1}$ and $\alpha_{2}$ are constants, and $x_{j 0}$ is the QCEW employment total for all establishments in domain $j$ for the month $t=0$. Taken together, Expressions (9) through (11) suggest that on a logarithmic scale, one may consider the variance model

$$
\ln \left\{V\left(\hat{y}_{j t}\right)\right\}=\ln (\Delta)+\alpha_{0} \ln (t)-\ln \left(n_{j t}\right)+\ln \left(\alpha_{1}\right)+\alpha_{2} \ln \left(x_{j 0}\right)+q_{j t}^{*}
$$

or in a slightly more general form,

$$
\ln \left\{V\left(\hat{y}_{j t}\right)\right\}=\gamma_{0}+\gamma_{1} \ln \left(n_{j t}\right)+\gamma_{2} \ln (t)+\gamma_{3} \ln \left(x_{j 0}\right)+q_{j t}^{*}
$$

where, for example, $\gamma_{0}=\ln (\Delta)+\ln \left(\alpha_{1}\right), \gamma_{1}=-1, \gamma_{2}=\alpha_{0}$ and $\gamma_{3}=\alpha_{2}$.

In addition, under some standard designs, the selected sample size $n_{j t}$ may be a function of variables related to $x_{j t}$. For example, under Neyman allocation (e.g., Cochran 1977, 99) $n_{j t}$ is proportional to $S_{j t} N_{j t}$ provided the domains were equal to individual strata, and so the log transformed Model (12) reduces to

$$
\ln \left(V_{p j t}\right)=\gamma_{0}+\gamma_{1} \ln \left(x_{j 0}\right)+\gamma_{2} \ln (t)+q_{j t}^{*}
$$

with appropriate redefinitions of the coefficients $\gamma_{0}, \gamma_{1}$ and $\gamma_{2}$. For Model (13), $X_{B_{j t}}=\left[\ln \left(x_{j 0}\right), \ln (t)\right]$. This model does not include any variables under the direct control of the designer, so $X_{A_{j t}}$ is empty. For the CES application, the domains were unions of several strata. Consequently, in preliminary work, we considered versions of Model (13) that included $X_{A_{j t}}=\ln \left(n_{j t}\right)$. However, our empirical results indicated that after inclusion of the predictor $\ln \left(x_{j 0}\right)$, the additional predictor $\ln \left(n_{j t}\right)$ provided very limited additional value. Consequently, our modeling work for this article centered on versions of Model (13). 
Finally, recall from Subsection 3.1 that GVF models often include the point estimator $\hat{\theta}_{j t}$ as a predictor. For the CES application, this would suggest inclusion of the population total estimators $\hat{y}_{j t}$. However, these estimators are strongly associated with the benchmark values $x_{j 0}$ which are already included in Model (13). Consequently, we did not include $\hat{y}_{j t}$ as an additional predictor in Model (13) for the CES data.

\subsection{Differences in Model Coefficients $\gamma$}

Model (13) was based on the assumption that the coefficient vector $\gamma$ was constant over all years and all domains. However, this assumption may not hold, for example, if the underlying terms $\Delta, \alpha_{0}, \alpha_{1}$, or $\alpha_{2}$ are not constant over years and domains. Consequently, we explored the possible heterogeneity of $\gamma$ over years and domains, respectively.

\subsubsection{Temporal Homogeneity}

To explore the temporal heterogeneity of $\hat{V}_{p j t}$, we divided years into two groups. National Bureau of Economic Research (NBER) declared the current recession starting December 2007. Moreover, the data from the BLS payroll employment site (http://data.bls.gov/ timeseries/CES0000000001?output_view =net_1mth) are generally consistent with the NBER recession timing. Consequently, we fit Model (13) separately for the years 2005-2007 and 2008-2010, respectively.

$$
\ln \left(V_{p j t}\right)=\left\{\begin{array}{lll}
\gamma_{10}+\gamma_{11} \ln \left(x_{j 0}\right)+\gamma_{12} \ln (t)+q_{j t}^{*} & \text { if } & \text { Year }=2005-2007 \\
\gamma_{20}+\gamma_{21} \ln \left(x_{j 0}\right)+\gamma_{22} \ln (t)+q_{j t}^{*} & \text { if } & \text { Year }=2008-2010
\end{array}\right.
$$

In addition, we tested for the homogeneity of coefficients across year groups, based on the null hypothesis

$$
H_{0}:\left(\gamma_{10}, \gamma_{11}, \gamma_{12}\right)=\left(\gamma_{20}, \gamma_{21}, \gamma_{22}\right)
$$

For this test, the Wald test statistic is:

$$
W=(A \hat{\gamma})^{\prime}\left\{A \hat{V}(\hat{\gamma}) A^{\prime}\right\}^{-1}(A \hat{\gamma})
$$

where $\gamma=\left(\gamma_{10}, \gamma_{11}, \gamma_{12}, \gamma_{20}, \gamma_{21}, \gamma_{22}\right), \hat{V}(\hat{\gamma})$ is a $6 \times 6$ design-based estimator of the covariance matrix of $\hat{\gamma}$ as described in Subsection 3.2 and

$$
A=\left(\begin{array}{rrrrrr}
1 & 0 & 0 & -1 & 0 & 0 \\
0 & 1 & 0 & 0 & -1 & 0 \\
0 & 0 & 1 & 0 & 0 & -1
\end{array}\right)
$$

Standard arguments adapted to the current case (e.g., Korn and Graubard 1990) indicate that $(W / d)\{(d-p+1) / p\}$ has approximately a noncentral $F$ distribution with $p$ and $(d-p+1)$ degrees of freedom and with noncentrality parameter $(A \gamma)^{\prime}\left\{A V(\gamma) A^{\prime}\right\}^{-1}(A \gamma)$ where $l=28$ is number of clusters (due to the presence of two groups of years intersected with 14 industries); $d=l-1=27$; and $p=3$ is number of rows in the contrast Matrix $A$.

Table 2 presents the resulting coefficient estimates, standard errors and test statistics. The separate blocks of rows in Table 2 correspond to separate model fits for variance 


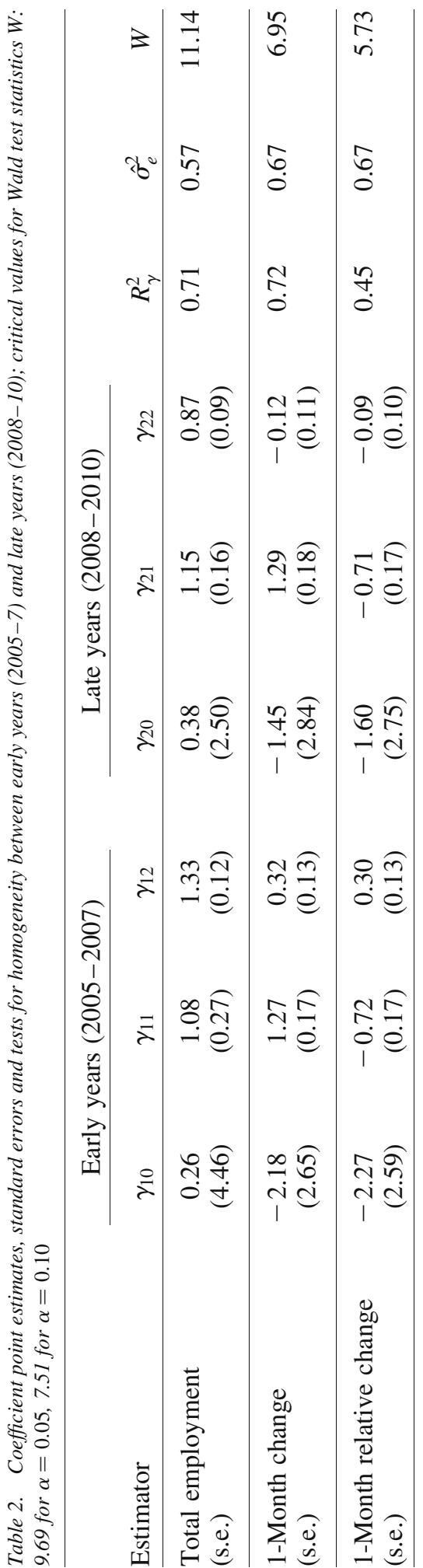


estimates $\hat{V}_{p j t}$ associated with total employment, one-month change and one-month relative change, respectively. Note especially the strong indications of statistically significant coefficients for $\ln \left(x_{j 0}\right)$ for each of the model fits. The coefficient estimates of $\ln \left(x_{j 0}\right)$ are positive for total employment and one-month change, reflecting the fact that larger values of $\ln \left(V_{p j t}\right)$ were generally associated with domains that had larger levels of employment and employment change.

For total employment, one-month change and one-month relative change estimators, the $W$ values are $11.14,6.95$ and 5.73, respectively. The cutoff points $\{d p /(d-p+1)\}$ $F_{0.05}\{p,(d-p+1)\}$ were 9.69 for $\alpha=0.05$ and 7.51 for $\alpha=0.10$. Note that the test statistic for total employment is much larger than both cutoff points. Thus, at conventional levels of significance, for the case of total employment, we reject the null hypothesis of equality of the GVF coefficients across the two groups of years. In addition, note that for total employment, the coefficient for the predictor $\ln (t)$ changes substantially between 2005-2007 $\left(\hat{\gamma}_{12}=1.33\right)$ and 2008-2010 $\left(\hat{\gamma}_{22}=0.87\right)$, relative to the magnitude of $\operatorname{se}\left(\hat{\gamma}_{12}\right)=0.12$. This illustrates the importance of carrying out empirical checks on the homogeneity of variance function models across years, rather than just assuming that the coefficients are constant.

\subsubsection{Cross-Sectional Homogeneity}

To explore the cross-sectional variability of $\hat{V}_{p j t}$, we fit Model (13) separately for domains in goods-producing and service-providing industries, respectively, which led to the model

$$
\ln \left(V_{p j t}\right)= \begin{cases}\gamma_{10}+\gamma_{11} \ln \left(x_{j 0}\right)+\gamma_{12} \ln (t)+q_{j t}^{*} & \text { if Goods (four industries) } \\ \gamma_{20}+\gamma_{21} \ln \left(x_{j 0}\right)+\gamma_{22} \ln (t)+q_{j t}^{*} & \text { if Services (ten industries) }\end{cases}
$$

In addition, we tested the null hypothesis $H_{0}:\left(\gamma_{10}, \gamma_{11}, \gamma_{12}\right)=\left(\gamma_{20}, \gamma_{21}, \gamma_{22}\right)$ using the Wald test statistic (15) where $l=14$ is number of clusters because there are two industry groups: one with four industries and the other with ten industries; $d=l-1=13$; and $p=3$ is number of rows in the contrast Matrix $A$. Table 3 presents the results of these analyses. As with Table 2, we have three sets of results for total employment, one-month change and one-month relative change, respectively. For estimators of total, one-month change and one-month relative change, the $W$ values were 15.94, 65.33 and 53.52, respectively. The cutoff points were 12.72 for $\alpha=0.05$, and 9.43 for $\alpha=0.10$.

Thus we have strong indication of differences in the Goods and Services coefficients for all three sets of estimators.

Finally, note that in both Table 2 and Table 3, the $R^{2}$ values for the total employment and one-month change were relatively strong (greater than 0.7 in each case). For the two GVF model fits for one-month relative change, the $R^{2}$ values were somewhat lower (0.45 and 0.49 , respectively).

\subsection{Evaluation of Sources of Variability in the CES Variance Estimators}

After evaluating the coefficient estimators $\hat{\gamma}$ for the CES data, we applied the diagnostic ideas outlined in Section 4. 


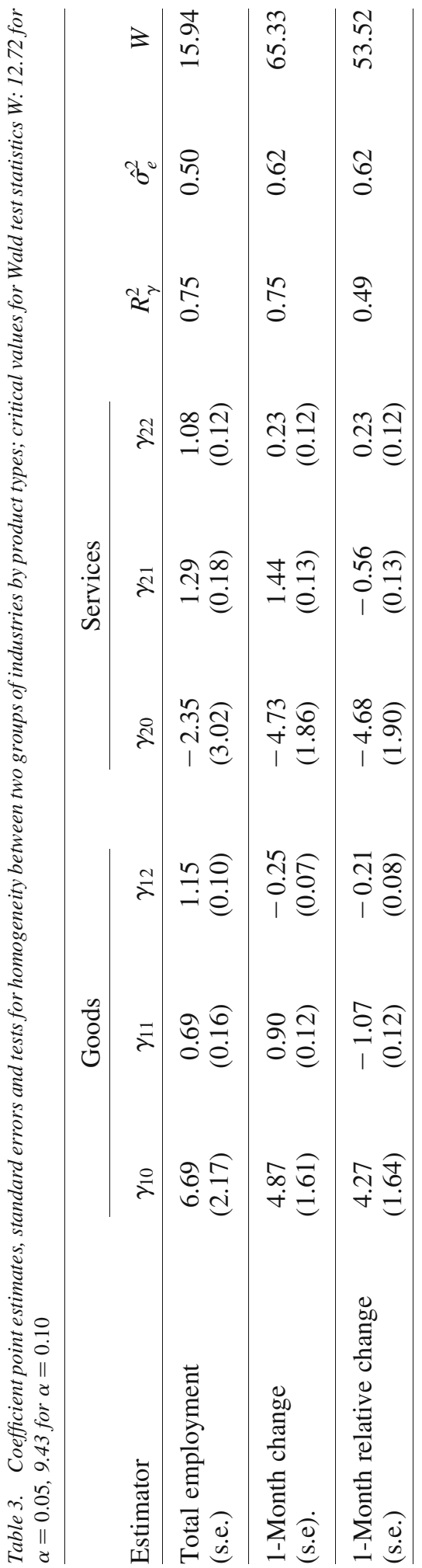


Table 4 presents results for full and partial model fits for the variance estimators $\hat{V}_{p j t}$ for total employment. In keeping with the results of Table 2, we allowed separate coefficients for the early years (2005-2007) and the late years (2008-2010), respectively. Note that in Table 4, in the full model fit for both early and late years, all coefficients (except for the intercept) are statistically significant at conventional $\alpha$ levels. In addition, $R^{2}=0.71,0.67$ and 0.05 for the full model fit, the fit with $\ln (t)$ omitted, and the fit with $\ln \left(x_{j 0}\right)$ omitted, respectively. In that sense, most of the explanatory power of Model (14) is attributable to the predictors $\ln \left(x_{j 0}\right)$. This also indicates that although the coefficient of $\ln (t)$ satisfies significance testing criteria at customary $\alpha$ levels, it does not contribute much power for prediction of $\ln \left(V_{j t}\right)$ as reflected in $R^{2}$ and $\sigma_{e}^{2}$ values. This illustrates the importance of using the diagnostics of Section 4 as complements to the coefficient testing idea from Section 3.

Tables 5 and 6 present related results for the variance estimators $\hat{V}_{p j t}$, associated with one-month change and one-month relative change, respectively. Table 5 displays patterns of statistical significance and $R^{2}$ results that are similar to those observed in Table 4, except that for the late years, the full model fit does not lead to statistically significant coefficients for the predictor $\ln (t)$. The results in Table 6 differ from those in Tables 4 and 5 in two notable ways. First, in the full-model fit, the estimates for $\gamma_{0}$ and $\gamma_{1}$ are negative in Table 6, but positive in Table 4. Second, the $R^{2}$ values in Table 6 are notably smaller than those in Tables 4 and 5 for the full model fit and the $\ln (t)$-omitted fits. Because the underlying point estimator for Table 6 is a ratio, one would not necessarily expect Table 6 to display the same pattern as observed for point estimators for totals and differences of totals as in Tables 4 and 5, respectively.

\subsection{Magnitude of Equation Error Variances}

To address issues (A) and (B) of Section 1, Subsection 5.2 developed methods for the identification of predictors $X_{j t}$ that account for some of the observed variability in the

Table 4. Total employment: coefficient estimates, inferential statistics and $R^{2}$ values for full-model and reduced-model fits

\begin{tabular}{|c|c|c|c|c|c|c|c|c|}
\hline \multirow[b]{3}{*}{ Model } & \multicolumn{3}{|c|}{ Early years $(2005-2007)$} & \multicolumn{3}{|c|}{ Late years $(2008-2010)$} & \multirow[b]{3}{*}{$R_{\gamma}^{2}$} & \multirow[b]{3}{*}{$\hat{\sigma}_{e}^{2}$} \\
\hline & Intercept & $\ln \left(x_{j 0}\right)$ & $\ln (t)$ & Intercept & $\ln \left(x_{j 0}\right)$ & $\ln (t)$ & & \\
\hline & $\begin{array}{c}\gamma_{0} \\
\text { (s.e.) } \\
\left(t_{\gamma_{0}}\right)\end{array}$ & $\begin{array}{c}\gamma_{1} \\
\text { (s.e.) } \\
\left(t_{\gamma_{1}}\right) \\
\end{array}$ & $\begin{array}{c}\gamma_{2} \\
\text { (s.e.) } \\
\left(t_{\gamma_{2}}\right)\end{array}$ & $\begin{array}{c}\gamma_{0} \\
\text { (s.e.) } \\
\left(t_{\gamma_{0}}\right)\end{array}$ & $\begin{array}{c}\gamma_{1} \\
\text { (s.e.) } \\
\left(t_{\gamma_{1}}\right) \\
\end{array}$ & $\begin{array}{c}\gamma_{2} \\
(\text { s.e. }) \\
\left(t_{\gamma_{2}}\right) \\
\end{array}$ & & \\
\hline Full & $\begin{array}{c}0.26 \\
(4.46) \\
(0.06)\end{array}$ & $\begin{array}{c}1.08 \\
(0.27) \\
(3.93)\end{array}$ & $\begin{array}{c}1.33 \\
(0.12) \\
(11.31)\end{array}$ & $\begin{array}{c}0.38 \\
(2.50) \\
(0.15)\end{array}$ & $\begin{array}{c}1.15 \\
(0.16) \\
(7.42)\end{array}$ & $\begin{array}{c}0.87 \\
(0.09) \\
(9.46)\end{array}$ & 0.71 & 0.57 \\
\hline $\begin{array}{l}\ln (t) \\
\text { omitted }\end{array}$ & $\begin{array}{c}3.66 \\
(4.35) \\
(0.84)\end{array}$ & $\begin{array}{l}1.08 \\
(0.27) \\
(3.93)\end{array}$ & $\begin{array}{l}- \\
- \\
-\end{array}$ & $\begin{array}{l}2.62 \\
(2.41) \\
(1.09)\end{array}$ & $\begin{array}{l}1.15 \\
(0.16) \\
(7.42)\end{array}$ & $\begin{array}{l}- \\
- \\
-\end{array}$ & 0.67 & 0.66 \\
\hline $\begin{array}{l}\ln \left(x_{j 0}\right) \\
\text { omitted }\end{array}$ & $\begin{array}{c}16.97 \\
(0.62) \\
(27.41)\end{array}$ & $\begin{array}{l}- \\
- \\
-\end{array}$ & $\begin{array}{c}1.33 \\
(0.12) \\
(11.31)\end{array}$ & $\begin{array}{c}18.21 \\
(0.48) \\
(38.05)\end{array}$ & $\begin{array}{l}- \\
- \\
-\end{array}$ & $\begin{array}{c}0.87 \\
(0.09) \\
(9.46)\end{array}$ & 0.05 & 1.89 \\
\hline
\end{tabular}


Table 5. One-month change: coefficient estimates, inferential statistics and $R^{2}$ values for full-model and reduced-model fits

\begin{tabular}{|c|c|c|c|c|c|c|c|c|}
\hline \multirow[b]{3}{*}{ Model } & \multicolumn{3}{|c|}{ Early years } & \multicolumn{3}{|c|}{ Late years } & \multirow[b]{3}{*}{$R_{\gamma}^{2}$} & \multirow[b]{3}{*}{$\hat{\sigma}_{e}^{2}$} \\
\hline & Intercept & $\ln \left(x_{j 0}\right)$ & $\ln (t)$ & Intercept & $\ln \left(x_{j 0}\right)$ & $\ln (t)$ & & \\
\hline & $\begin{array}{c}\gamma_{0} \\
(s . e .) \\
\left(t_{\gamma_{0}}\right) \\
\end{array}$ & $\begin{array}{c}\gamma_{1} \\
(s . e .) \\
\left(t_{\gamma_{1}}\right)\end{array}$ & $\begin{array}{c}\gamma_{2} \\
(\text { s.e. }) \\
\left(t_{\gamma_{2}}\right)\end{array}$ & $\begin{array}{c}\gamma_{0} \\
(s . e .) \\
\left(t_{\gamma_{0}}\right) \\
\end{array}$ & $\begin{array}{c}\gamma_{1} \\
(s . e .) \\
\left(t_{\gamma_{1}}\right)\end{array}$ & $\begin{array}{c}\gamma_{2} \\
(s . e .) \\
\left(t_{\gamma_{2}}\right)\end{array}$ & & \\
\hline Full & $\begin{array}{c}-2.18 \\
(2.65) \\
(-0.82)\end{array}$ & $\begin{array}{c}1.27 \\
(0.17) \\
(7.32)\end{array}$ & $\begin{array}{c}0.32 \\
(0.13) \\
(2.44)\end{array}$ & $\begin{array}{c}-1.45 \\
(2.84) \\
(-0.51)\end{array}$ & $\begin{array}{l}1.29 \\
(0.18) \\
(7.20)\end{array}$ & $\begin{array}{c}-0.12 \\
(0.11) \\
(-1.16)\end{array}$ & 0.72 & 0.67 \\
\hline $\begin{array}{l}\ln (t) \\
\text { omitted }\end{array}$ & $\begin{array}{c}-1.37 \\
(2.74) \\
(-0.50)\end{array}$ & $\begin{array}{c}1.27 \\
(0.17) \\
(7.32)\end{array}$ & $\begin{array}{l}- \\
- \\
-\end{array}$ & $\begin{array}{c}-1.76 \\
(2.80) \\
(-0.63)\end{array}$ & $\begin{array}{c}1.29 \\
(0.18) \\
(7.20)\end{array}$ & $\begin{array}{l}- \\
- \\
-\end{array}$ & 0.72 & 0.68 \\
\hline $\begin{array}{l}\ln \left(x_{j 0}\right) \\
\text { omitted }\end{array}$ & $\begin{array}{c}17.53 \\
(0.39) \\
(44.96)\end{array}$ & $\begin{array}{l}- \\
- \\
-\end{array}$ & $\begin{array}{c}0.32 \\
(0.13) \\
(2.44)\end{array}$ & $\begin{array}{c}18.43 \\
(0.36) \\
(50.98)\end{array}$ & $\begin{array}{l}- \\
- \\
-\end{array}$ & $\begin{array}{c}-0.12 \\
(0.11) \\
(-1.16)\end{array}$ & 0.01 & 2.41 \\
\hline
\end{tabular}

estimators $\hat{V}_{p j t}$. Furthermore, Sections 4 and 5.3 used standard regression diagnostics to evaluate the properties of variability in $\ln \left(\hat{V}_{p j t}\right)$ that is attributable to specific predictors, $X$.

To address issue $(\mathrm{C})$, this section will consider the variability of the residual terms $\hat{q}_{j t}^{*}=\ln \left(\hat{V}_{p j t}\right)-X_{j t} \hat{\gamma}$. In particular, we address issue (C) by exploring the extent to which the variances of the residuals $\hat{q}_{j t}^{*}$ may vary across industry, employment size at benchmark month, or month.

Figure 6 presents a scatter plot of these monthly residuals for total employment against the predicted values with separate plotting symbols for industries that are goods producing (1-4) and service providing (5-14), respectively. To explore this further, Table 7 presents

Table 6. One-month relative change: coefficient estimates, inferential statistics and $R^{2}$ values for full-model and reduced-model fits

\begin{tabular}{|c|c|c|c|c|c|c|c|c|}
\hline \multirow[b]{3}{*}{ Model } & \multicolumn{3}{|c|}{ Early years } & \multicolumn{3}{|c|}{ Late years } & \multirow[b]{3}{*}{$R_{\gamma}^{2}$} & \multirow[b]{3}{*}{$\hat{\sigma}_{e}^{2}$} \\
\hline & Intercept & $\ln \left(x_{j 0}\right)$ & $\ln (t)$ & Intercept & $\ln \left(x_{j 0}\right)$ & $\ln (t)$ & & \\
\hline & $\begin{array}{c}\gamma_{0} \\
(s . e .) \\
\left(t_{\gamma_{0}}\right)\end{array}$ & $\begin{array}{c}\gamma_{1} \\
(s . e .) \\
\left(t_{\gamma_{1}}\right)\end{array}$ & $\begin{array}{c}\gamma_{2} \\
(s . e .) \\
\left(t_{\gamma_{2}}\right) \\
\end{array}$ & $\begin{array}{c}\gamma_{0} \\
(s . e .) \\
\left(t_{\gamma_{0}}\right)\end{array}$ & $\begin{array}{c}\gamma_{1} \\
(s . e .) \\
\left(t_{\gamma_{1}}\right)\end{array}$ & $\begin{array}{c}\gamma_{2} \\
(s . e .) \\
\left(t_{\gamma_{2}}\right)\end{array}$ & & \\
\hline Full & $\begin{array}{c}-2.27 \\
(2.59) \\
(-0.88)\end{array}$ & $\begin{array}{c}-0.72 \\
(0.17) \\
(-4.27)\end{array}$ & $\begin{array}{c}0.30 \\
(0.13) \\
(2.38)\end{array}$ & $\begin{array}{c}-1.60 \\
(2.75) \\
(-0.58)\end{array}$ & $\begin{array}{c}-0.71 \\
(0.17) \\
(-4.08)\end{array}$ & $\begin{array}{c}-0.09 \\
(0.10) \\
(-0.84)\end{array}$ & 0.45 & 0.67 \\
\hline $\begin{array}{l}\ln (t) \\
\text { omitted }\end{array}$ & $\begin{array}{c}-1.50 \\
(2.68) \\
(-0.56)\end{array}$ & $\begin{array}{l}-0.72 \\
(0.17) \\
(-4.27)\end{array}$ & $\begin{array}{l}- \\
- \\
-\end{array}$ & $\begin{array}{l}-1.82 \\
(2.72) \\
(-0.67)\end{array}$ & $\begin{array}{c}-0.71 \\
(0.17) \\
(-4.08)\end{array}$ & $\begin{array}{l}- \\
- \\
-\end{array}$ & 0.45 & 0.67 \\
\hline $\begin{array}{l}\ln \left(x_{j 0}\right) \\
\text { omitted }\end{array}$ & $\begin{array}{c}-13.52 \\
(0.42) \\
(-32.19)\end{array}$ & $\begin{array}{l}- \\
- \\
-\end{array}$ & $\begin{array}{c}0.30 \\
(0.13) \\
(2.38)\end{array}$ & $\begin{array}{c}-12.57 \\
(0.42) \\
(-30.00)\end{array}$ & $\begin{array}{l}- \\
- \\
-\end{array}$ & $\begin{array}{c}-0.09 \\
(0.10) \\
(-0.84)\end{array}$ & 0.00 & 1.21 \\
\hline
\end{tabular}




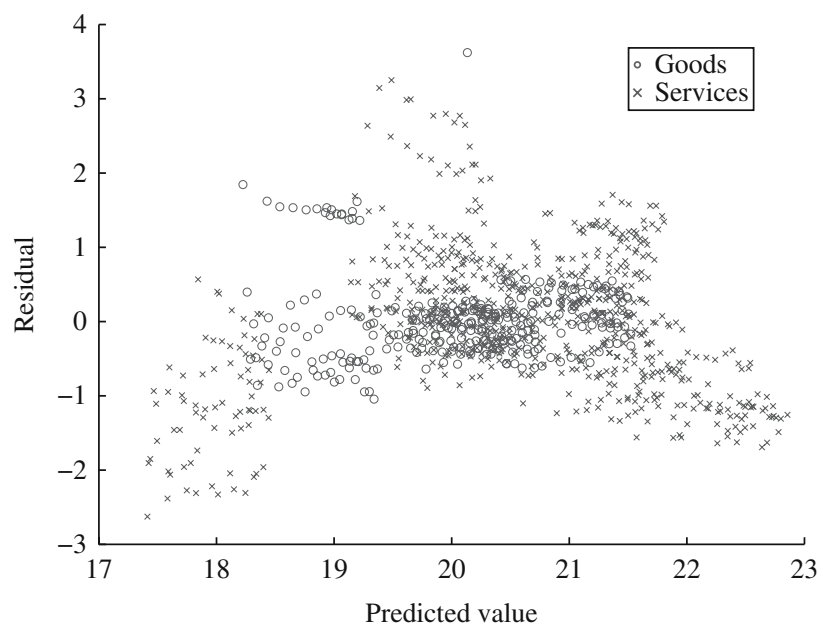

Fig. 6. Scatter plot of log-scale residuals $\hat{q}_{j t}^{*}$ against predicted values $X_{j t} \hat{\gamma}$ for the variance of total employment

selected sample quantiles of these residuals for goods-producing and service-providing industries, respectively, based on data from 2005-2010. Note especially that for each of total employment, one-month change, and one-month relative change, the interquartile range (IQR) for goods is somewhat larger than the IQR for services. However, the difference between the 99th percentile and the first percentile is larger for services than for goods with total employment, and are approximately equal with one-month change and one-month relative change.

In addition, we fit the models,

$$
\left(\hat{q}_{j t}^{*}\right)^{2}= \begin{cases}\omega_{G 0}+\omega_{G 1} \ln \left(x_{j 0}\right)+\omega_{G 2} \ln (t) & \text { if } j \in \text { Goods } \\ \omega_{S 0}+\omega_{S 1} \ln \left(x_{j 0}\right)+\omega_{S 2} \ln (t) & \text { if } j \in \text { Services }\end{cases}
$$

and tested $H_{0}:\left(\omega_{G 0}, \omega_{G 1}, \omega_{G 2}\right)=\left(\omega_{S 0}, \omega_{S 1}, \omega_{S 2}\right)$ using estimators and test statistics similar to those developed in Subsection 5.2.

Table 8 presents the resulting coefficient estimates, standard errors and test statistics. Note that the Wald tests do not reject the null hypothesis of no differences for the total employment, one-month change and one-month relative change analyses. However, for one-month change and one-month relative change, $t$-tests on individual coefficients are fairly distinct for the "Goods" and "Services" models, respectively. In particular, for the "Goods" analyses, the coefficients for $\ln \left(x_{j 0}\right)$ are not significant for these two cases; and for the "Services" analyses, the coefficients for $\ln \left(x_{j 0}\right)$ are significant for the corresponding two cases.

Reviewers of an earlier form of this article noted that a version of Figure 6 displays curvature for the service-providing industries. To address this, we fit an alternative form of Model (13) that included the predictor $\left\{\ln \left(x_{j 0}\right)\right\}^{2}$. Table 9 presents results for the model

$$
\ln \left(\hat{V}_{p j t}\right)= \begin{cases}\gamma_{10}+\gamma_{11} \ln \left(x_{j 0}\right)+\gamma_{111}\left\{\ln \left(x_{j 0}\right)\right\}^{2}+\gamma_{12} \ln (t)+q_{j t}^{*} & \text { if Year }=2005-2007 \\ \gamma_{20}+\gamma_{21} \ln \left(x_{j 0}\right)+\gamma_{211}\left\{\ln \left(x_{j 0}\right)\right\}^{2}+\gamma_{22} \ln (t)+q_{j t}^{*} & \text { if Year }=2008-2010\end{cases}
$$




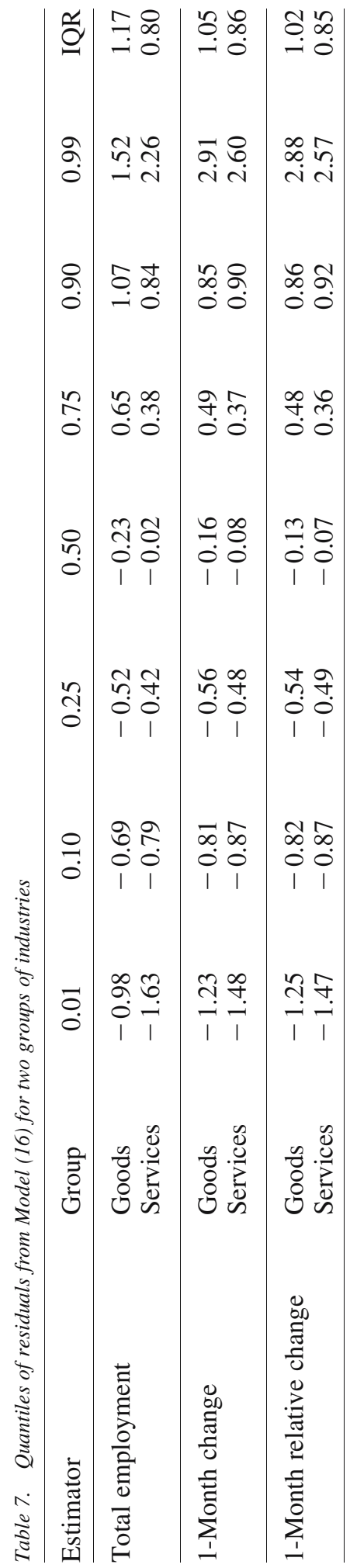




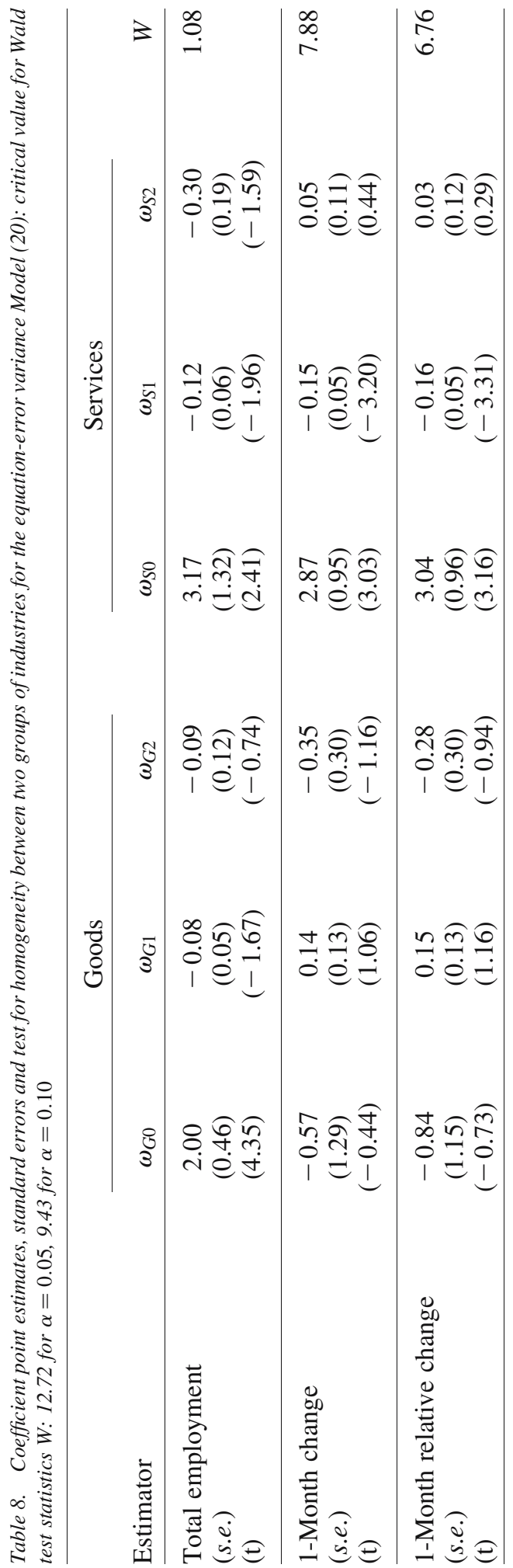




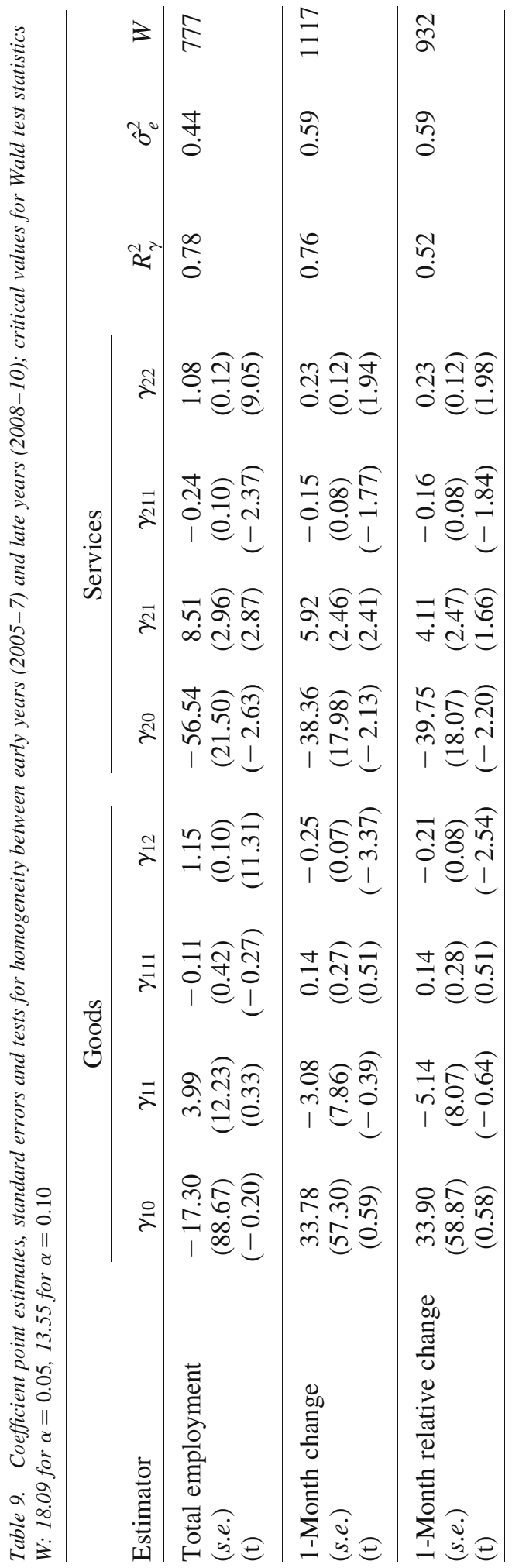


A plot of the residuals from Model (18) against the predicted values produced by Model (18) did not display the curvature pattern observed in Figure 6. However, in comparison with the results in Table 3 for Model (16), Table 9 indicates that for Model (18), inclusion of the squared predictor to modest changes in the values of $\sigma_{e}^{2}(0.50 \mathrm{vs} 0.44)$ and $R^{2}(0.75$ vs 0.78$)$. In addition, for goods-producing industries, the $t$-statistics reported for the coefficient $\gamma_{111}$ of $\left\{\ln \left(x_{j 0}\right)\right\}^{2}$ are not significant at conventional levels of significance; however, for service-providing industries, the $t$-statistics reported for the coefficients $\gamma_{211}$ equal $-2.37,-1.77$ and -1.84 for total employment, one-month change and one-month relative change respectively. Use of an additional regressor $\ln \left(n_{j t}\right)$ was explored through analyses that are not detailed in the current text; inclusion of this regressor did not produce notable changes in the analyses.

In summary, Subsections 5.2 and 5.3 indicated that much of the observed variability in the $\ln \left(\hat{V}_{p j t}\right)$ values may be attributed to variability in the conditional-expectation structure described by the regression Model (13). In addition, those sections indicated importance of testing for homogeneity of model fit across different temporal and cross-sectional groups. The current section indicates that the patterns of residual variability (reflected in the variances of the equation error terms $q_{j t}^{*}$ ) differ substantially between goodsproducing and service-providing industries, and in some cases may be associated with the predictors $\ln \left(x_{j 0}\right)$.

\section{Discussion}

Historically, survey organizations have developed generalized variance function models based on relatively broad concepts like commonality in design and population features. This article complements these previous approaches by using formal significance procedures to test for homogeneity of GVF coefficients across groups of estimators; by using regression diagnostics to evaluate the impact of adding particular predictors; and by using models for the variances of the equation errors in GVF models. The CES application presented in Sections 2 and 5 illustrated the main ideas of this article, with special emphasis on comparison of models across years (2005-2007 vs 2008-2010) and across industry groups (goods-producing vs service-providing).

One could consider several extensions of the ideas developed here. First, this article used the assumption that the rescaled variance estimators $V_{p j t}^{-1} d_{j t} \hat{V}_{p j t}$ followed a chisquare distribution on $d_{j t}$ degrees of freedom with values of $d_{j t}$ that were large (over 100). This was appropriate for the national-level analyses considered here. It would be of interest to extend the current work to state and local area analyses; for some of those analyses, the effective degrees of freedom $d_{j t}$ may be relatively small. In addition, one could consider alternative approaches under which scaled forms of $V_{p j t}^{-1} \hat{V}_{p j t}$ followed a heavy-tailed distribution, for example, a contaminated chi-square or contaminated lognormal. These alternatives may be of special interest for cases in which the underlying data may be subject to outliers.

Second, one could consider versions of Models (2) and (3) that directly incorporate finite population corrections (fpc). This would be of interest primarily in applications for which some strata have substantial sampling fractions, and for which explicit inclusion of fpc terms may lead to substantial improvements in the GVF model fit. For cases in which 
an estimator $\hat{\theta}_{j t}$ is based on data from a single stratum (as is the case for some types of domain estimation), explicit inclusion of a finite population correction term leads to an adjustment of the intercept terms in a logarithm scale fit of Model (3). For other cases, inclusion of a finite population correction leads to more complex adjustments that are beyond the scope of the current work.

Third, in keeping with the comments in Subsection 3.2, one could consider weighted least squares (WLS) or generalized least squares (GLS) point estimators of the coefficient vectors $\gamma$. These alternatives would be of special interest in cases for which ordinary least squares residual plots displayed patterns of heteroscedasticity that were more severe than the pattern in Figure 6 for the log-transformed fit. Under the alternative models described in the previous paragraph, it would be of special interest to explore conditions under which GLS estimators of $\gamma$ are more efficient than the ordinary least squares estimators used in this article, to develop variance estimators for these GLS point estimators, and to evaluate properties of the GLS estimators under violation of the abovementioned model assumptions.

Fourth, the proposed parametric GVF model in this article assumes that the model is fully described by a very small set of parameters. However, for other applications, the relationships between sampling variances and predictor variables may follow patterns that require more complex models with a larger number of parameters. Semiparametric analysis may provide a flexible tool for studying the dependence of a variable of interest on auxiliary information, without constraining the dependence to a fixed form with few parameters. It would be of interest to extend our model to the semiparametric setting.

\section{References}

Binder, D.A. 1983. "On the Variances of Asymptotically Normal Estimators from Complex Surveys.” International Statistical Review 51: 279-292.

Butani, S., G. Stamas, and M. Brick. 1997. "Sample Redesign for the Current Employment Statistics Survey." In Proceedings of the Section on Survey Research Methods: American Statistical Association, August 10-14, 1997. 517-522. Available at: http://www.amstat.org/sections/SRMS/Proceedings/papers/1997088.pdf (accessed May 2014).

Cho, M.J., J.L. Eltinge, J. Gershunskaya, and L. Huff. 2002. "Evaluation of the Predictive Precision of Generalized Variance Functions in the Analysis of Complex Survey Data." In Proceedings of the Section on Survey Research Methods: American Statistical Association, August 11-15, 2002. 534-539. Available at http://www.amstat.org/ sections/SRMS/Proceedings/y2002/Files/JSM2002-000845.pdf (accessed May 2014).

Cho, M., J.L. Eltinge, J. Gershunskaya, and L. Huff. 2014. "Evaluation of Generalized Variance Functions in the Analysis of Complex Survey Data." Journal of Official Statistics 30: 63-90.

Cochran, W.G. 1977. Sampling Techniques, (Third Edition). New York: John Wiley and Sons.

Copeland, K.R., and R. Valliant. 2007. "Imputing for Late Reporting in the U.S. Current Employment Statistics Survey.” Journal of Official Statistics 23: 69-90. 
Draper, N.R., and H. Smith. 1998. Applied Regression Analysis, (Third Edition). New York: John Wiley and Sons.

Fuller, W.A. 1987. Measurement Error Models. New York: John Wiley and Sons.

Gershunskaya, J., and P. Lahiri. 2005. "Variance Estimation for Domains in the U.S. Current Employment Statistics Program.” In Proceedings of the Section on Survey Research Methods: American Statistical Association, August 7-11, 2005. 3044-3051. Available at: http://www.amstat.org/sections/SRMS/Proceedings/y2005/Files/ JSM2005-000411.pdf (accessed May 2014).

Johnson, E.G., and B.F. King. 1987. "Generalized Variance Functions for a Complex Sample Survey." Journal of Official Statistics 3: 235-250.

Judkins, D.R. 1990. "Fay's Method for Variance Estimation.” Journal of Official Statistics 6: $223-239$.

Kish, L. 1995. "Methods for Design Effects.” Journal of Official Statistics 11: 55-77.

Korn, E.L., and B.I. Graubard. 1990. "Simultaneous Testing of Regression Coefficients with Complex Survey Data: Use of Bonferroni $t$ statistics." The American Statistician 44: 270-276.

O’Malley, A.J., and A.M. Zaslavsky. 2005. "Variance-Covariance Functions for Domain Means of Ordinal Survey Items." Survey Methodology 31: 169-182.

Park, I., and H. Lee. 2004. "Design Effects for Weighted Mean and Total Estimators Under Complex Survey Sampling." Survey Methodology 30: 183-193.

U.S. Bureau Of Labor Statistics. 2011. BLS Handbook of Methods. Washington, DC: U.S. Department of Labor. Available at: http://www.bls.gov/opub/hom/pdf/homch2.pdf (accessed May 2014).

Valliant, R. 1987. "Generalized Variance Functions in Stratified Two-Stage Sampling." Journal of American Statistical Association 82: 499-508.

Valliant, R., and K. Rust. 2010. "Degrees of Freedom Approximation and Rulesof-Thumb." Journal of Official Statistics 26: 585-602.

Werking, G. 1997. "Overview of the CES Redesign.” In Proceedings of the Section on Survey Research Methods: American Statistical Association, August 10-14, 1997. 512-516. Available at: http://www.amstat.org/sections/SRMS/Proceedings/papers/ 1997 087.pdf (accessed May 2014).

Wolter, K.M. 2007. Introduction to Variance Estimation, (Second Edition). New York: Springer-Verlag.

Received December 2012

Revised May 2014

Accepted July 2014 